\title{
Perfil de pesquisadores científicos das regiões nordeste e sudeste do Brasil
}

\author{
Profile of scientific researchers from the northeast and southeast regions of Brazil \\ Perfil de investigadores científicos de las regiones noreste y sureste de Brasil
}

Recebido: 30/01/2022 | Revisado: 04/02/2022 | Aceito: 11/02/2022 | Publicado: 17/02/2022

\author{
Francisco das Chagas Araújo Sousa \\ ORCID: https://orcid.org/0000-0001-7244-9729 \\ Universidade Estadual do Piauí, Brasil \\ E-mail: franciscoaraujo@ccs.uespi.br \\ Jassiara de Sousa Nascimento da Luz \\ ORCID: https://orcid.org/0000-0003-0993-8065 \\ Universidade Estadual do Piauí, Brasil \\ E-mail: jassiarasousa17@hotmail.com \\ Láyla Lorrana de Sousa Costa \\ ORCID: https://orcid.org/0000-0003-0533-0116 \\ Universidade Estadual do Piauí, Brasil \\ E-mail: laylalorranasousa@gmail.com \\ Franciscco Aragão de Sousa Neto \\ ORCID: https://orcid.org/0000-0003-2060-1324 \\ Universidade Estadual do Piauí, Brasil \\ E-mail: aragaoneto24@gmail.com \\ Tayrine Helen Marques do Nascimento \\ ORCID: https://orcid.org/0000-0002-1848-3085 \\ Universidade Estadual do Piauí, Brasil \\ E-mail: tayrinehelen10@gmail.com \\ Wellen Andreina dos Santos Silva \\ ORCID: https://orcid.org/0000-0001-9062-8958 \\ Universidade Estadual do Piauí, Brasil \\ E-mail: wellenandreina123@gmail.com \\ Maria Hannele Morais Barreiros \\ ORCID: https://orcid.org/0000-0002-9759-5404 \\ Universidade Estadual do Piauí, Brasil \\ E-mail: mariabarreiros@aluno.uespi.br \\ Ana Karla de Sousa Silva \\ ORCID: https://orcid.org/0000-0001-5401-4764 \\ Universidade Estadual do Piauí, Brasil \\ E-mail: anafisioterapia38@gmail.com \\ Luiz Henrique Carvalho Silva \\ ORCID: https://orcid.org/0000-0002-8128-1494 \\ Universidade Estadual do Piauí, Brasil \\ E-mail: luizkarvalho37@gmail.com \\ Sabrina Mendes Silva \\ ORCID: https://orcid.org/0000-0003-4267-7610 \\ Universidade Estadual do Piauí, Brasil \\ E-mail: sabrinamendes324@gmail.com \\ Wenderson Costa da Silva \\ ORCID: https://orcid.org/0000-0001-6031-9775 \\ Universidade Estadual do Maranhão, Brasil \\ E-mail: wendersoncosta09@hotmail.com
}

\section{Resumo}

Nas últimas décadas, foi observado que nas áreas de medicina, física, ciências humanas, sociais e engenharias houve um notável aumento na produção científica. A pesquisa é importante para o fomento da produção científica especialmente no Nordeste, região em que profissionais encontram pouco acesso à programas de pós-graduação. O objetivo desse estudo foi escrever o perfil de Pesquisadores de Pós-Graduação de Instituições Públicas nas regiões Nordeste e Sudeste. Trata-se de uma pesquisa de campo de natureza básica, do tipo longitudinal retrospectiva documental com uma abordagem quantitativa dos dados. A coleta de dados teve duração de 4 meses e foi feita diretamente pelos pesquisadores, através de acessos à plataforma Lattes do CNPq na seção de Dados e Estatísticas. Observou-se que a produção cientifica é fortemente concentrada na região sudeste do Brasil, tanto no mestrado como doutorado. Os programas de doutorado também vêm crescendo de forma significativa no Brasil, porém ainda é menor que os programas de mestrado. Os dados mostram que o Brasil enfrenta diversos desafios no tocante ao desenvolvimento e à consolidação de seu Sistema Nacional de Inovação. Dentre eles, destacam-se as necessidades de 
incentivo às atividades de Pesquisa e Desenvolvimento. Os resultados da pesquisa demonstram claramente a existência de desigualdades no quantitativo da produção científica nas regiões. Destacam-se as necessidades de incentivo às atividades de Pesquisa e Desenvolvimento nas regiões para reverter essas desigualdades.

Palavras-chave: Pesquisa; Educação de pós-graduação; Avaliação educacional; Brasil; Indicadores de produção científica.

\begin{abstract}
In recent decades, it has been observed that in the areas of medicine, physics, humanities, social sciences and engineering there has been a notable increase in scientific production. Research is important to foster scientific production, especially in the Northeast, a region where well-known professionals have little access to graduate programs. The aim of this study was to write the profile of Postgraduate Researchers from Public Institutions in the Northeast and Southeast regions. It is a field research of basic nature, of the documentary retrospective type with a quantitative approach to the data. Data collection lasted 4 months and was carried out directly by the researchers, through access to the CNPq Lattes platform in the Data and Statistics section. It was observed that scientific production is strongly concentrated in the southeast region of Brazil, both in master's and doctoral programs. Doctoral programs also increase the form of growth in Brazil, but it is still smaller than master's programs. The data presented show that Brazil faces several challenges with regard to the development and consolidation of its National Innovation System. Among them, they stand out as a need to encourage Research and Development activities. The research results clearly demonstrate the existence of inequalities in the quantity of scientific production in the regions. They stand out as a need to encourage Research and Development activities in the regions to reverse these inequalities.
\end{abstract}

Keywords: Research; Graduate education; Educational assessment; Brazil; Scientific production indicators.

\title{
Resumen
}

En las últimas décadas se ha observado que en las áreas de medicina, física, humanidades, ciencias sociales e ingeniería se ha producido un notable incremento de la producción científica. La investigación es importante para fomentar la producción científica, especialmente en el Nordeste, una región donde los profesionales reconocidos tienen poco acceso a los programas de posgrado. El objetivo de este estudio fue redactar el perfil de Investigadores de Posgrado de Instituciones Públicas de las regiones Noreste y Sudeste. Se trata de una investigación de campo de carácter básico, de tipo documental retrospectivo con un enfoque cuantitativo de los datos. La recogida de datos tuvo una duración de 4 meses y fue realizada directamente por los investigadores, mediante el acceso a la plataforma $\mathrm{CNPq}$ Lattes en el apartado de Datos y Estadísticas. Se observó que la producción científica está fuertemente concentrada en la región sureste de Brasil, tanto en programas de maestría como de doctorado. Los programas de doctorado también aumentan la forma de crecimiento en Brasil, pero aún es menor que los programas de maestría. Los datos presentados muestran que Brasil enfrenta varios desafíos en cuanto al desarrollo y consolidación de su Sistema Nacional de Innovación. Entre ellos, destacan como una necesidad de incentivar las actividades de Investigación y Desarrollo. Los resultados de la investigación demuestran claramente la existencia de desigualdades en la cantidad de producción científica en las regiones. Destacan como una necesidad de incentivar las actividades de Investigación y Desarrollo en las regiones para revertir estas desigualdades.

Palabras clave: Investigación; Educación de postgrado; Evaluación educativa; Brasil; Indicadores de producción científica.

\section{Introdução}

Nas últimas décadas, foi observado que nas áreas de medicina, física, ciências humanas, sociais e engenharias houve um notável aumento na produção científica, comprovado pelo número de publicações no Institute for Scientific Information e no SciELO (Mendes et al., 2010).

Segundo Péret e Lima (2003) o desenvolvimento da pesquisa apresenta importante papel para a geração de novos conhecimentos, novas tecnologias e para o desenvolvimento do espírito crítico e reflexivo na formação acadêmica do profissional. Na área médica, foi visto que a ampliação de programas de pós-graduação e formação de novos grupos de pesquisa foi responsável por parte do crescimento de artigos científicos (Barral \& Barral-Netto, 2008).

O investimento do setor público também é fator importante no crescimento da produção científica brasileira, tanto na qualificação de profissionais como na infraestrutura de universidade e institutos de pesquisa (Helene \& Ribeiro, 2011).

No âmbito da evolução recente da produção científica mundial, observa-se que seu crescimento acelerado é acompanhado pelo aumento da colaboração entre os pesquisadores. No Brasil, se verifica enorme heterogeneidade espacial das 
atividades de pesquisa científica, onde o padrão regional da distribuição das publicações e dos pesquisadores é altamente concentrado na região Sudeste, com destaque às capitais dos estados (Sidone et al., 2016).

Os dados provenientes da Plataforma Lattes permitem que a pesquisa seja realizada de forma clara através da organização geográfica. A pesquisa é importante para o fomento da produção científica especialmente no Nordeste, região em que profissionais encontram pouco acesso à programas de pós-graduação.

Diante do exposto esteve estudo teve como objetivo geral escrever o perfil de Pesquisadores de Pós-Graduação de Instituições Públicas nas regiões Nordeste e Sudeste. E especificamente identificar a quantidade de mestres e doutores da área da saúde dos estados da região Nordeste; identificar a quantidade de mestres e doutores da área da saúde dos estados da região Sudeste; e comparar a quantidade de mestres e doutores de outras áreas com os da área da saúde.

\section{Metodologia}

Trata-se de uma pesquisa de campo de natureza básica, do tipo longitudinal retrospectiva documental com uma abordagem quantitativa do perfil da pós-graduação e grupos de pesquisas no Brasil.

A pesquisa descritiva utiliza dados dos levantamentos e caracteriza-se por hipóteses especulativas que não especificam relações de causalidade, tendo como principal objetivo a descrição das características de determinada população (Aaker et al., 2004).

A pesquisa quantitativa tem o intuito de validar as hipóteses frente ao uso de dados estruturados, estatísticos, com análise de um grande número de casos representativos, recomendando um curso final da ação. Esta pesquisa quantifica os dados e generaliza os resultados da amostra (Mattar, 2001).

A coleta de dados teve duração de 4 meses e foi feita diretamente pelos pesquisadores, através de acessos à plataforma Lattes do CNPq na seção de Dados e Estatísticas. Os critérios de inclusão foram todos os dados presentes na plataforma que se referem à categoria de pós-graduação mestrado e doutorado, que sejam de instituições de ensino públicas e das regiões Nordeste e Sudeste do Brasil, exclui-se dados que não estiverem na plataforma e os que não atenderem a esses quesitos.

O presente estudo trata-se de uma pesquisa documental na qual foi utilizada apenas fontes de domínio público, dessa forma não necessitou de aprovação do Comitê de Ética em Pesquisa para o seu desenvolvimento. Todavia, os cuidados éticos na coleta, análise e interpretação dos resultados de forma anônima foram seguidos rigorosamente.

Os benefícios relacionados à pesquisa foram relevantes à obtenção do conhecimento sobre o assunto e consequente estímulo a programas de pós-graduação, já que ao se deparar com os resultados, uma discussão será aberta e haverá promoção de educação e informação. Tal pesquisa pode servir de referência ou instigar novas pesquisas sobre o assunto. Os dados foram organizados e tabulados utilizando o Microsoft Excel versão 2010 para Windows.

\section{Resultados e Discussão}

Poucas são as pesquisas com os pesquisadores como sujeitos, a pesquisa atual buscou identificar a quantidade de pesquisadores das regiões nordeste e sudeste do Brasil e compara-las.

No Gráfico 1,observa-se que a produção cientifica é fortemente concentrada na região sudeste do Brasil, tanto no mestrado como doutorado. 
Research, Society and Development, v. 11, n. 3, e16611326334, 2022

(CC BY 4.0) | ISSN 2525-3409 | DOI: http://dx.doi.org/10.33448/rsd-v11i3.26334

Gráfico 1. Dados gerais de pesquisadores de ciências da saúde. Teresina, PI, Brasil, 2021.

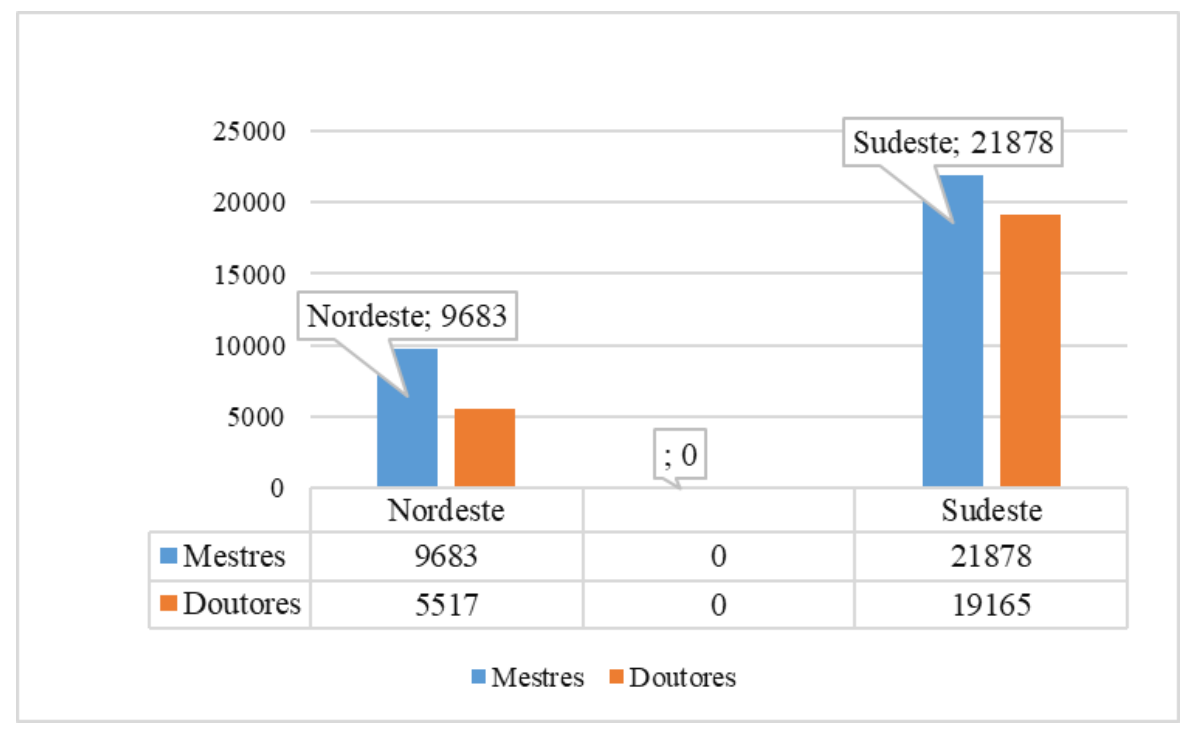

Fonte: $\mathrm{CNPq}(2021)$.

No Gráfico 2, foram tabelados dados para compararmos a quantidade de mestres e doutores de outras áreas com os da área da saúde, nas régios nordeste e sudeste.

Gráfico 2. Dados gerais de pesquisadores das demais áreas. Teresina, PI, Brasil, 2021.

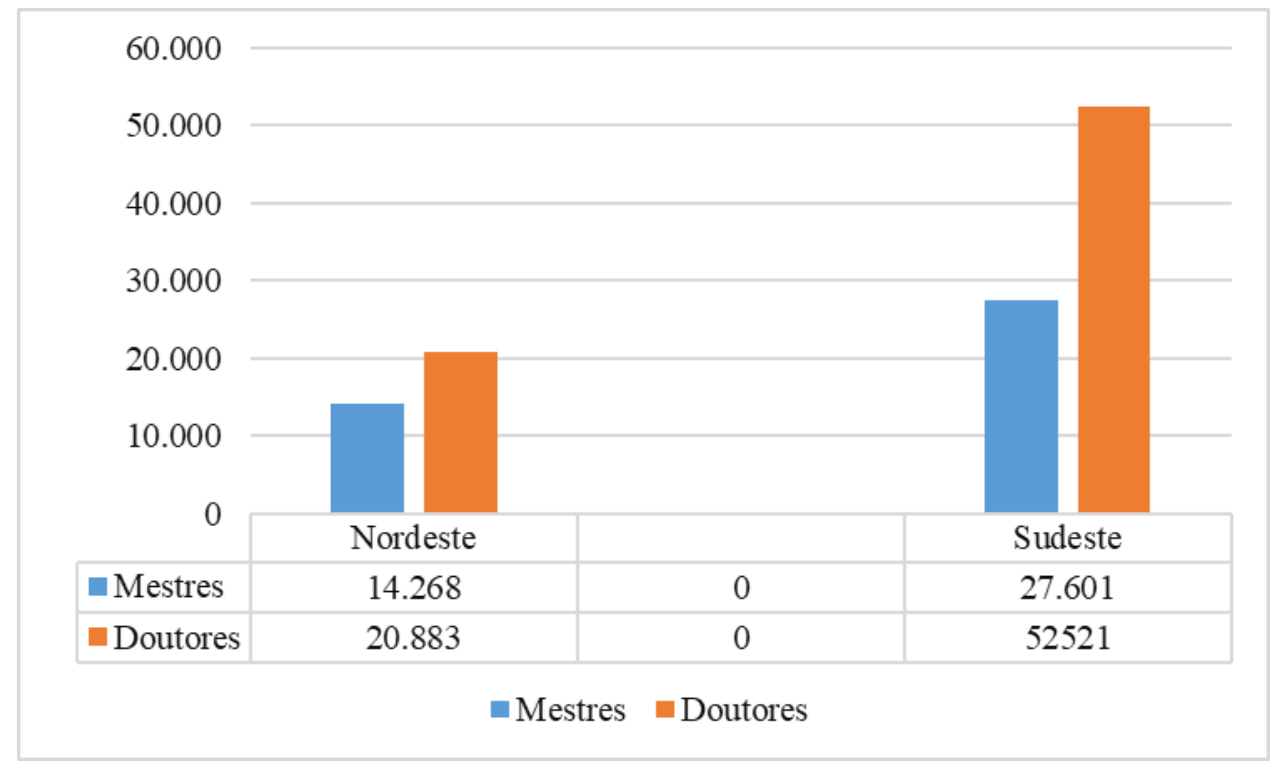

Fonte: CNPq (2021). 
Gráfico 3. Produção cientifica de ciencias da saude no nordeste e sudeste. Teresina, PI, Brasil, 2021.

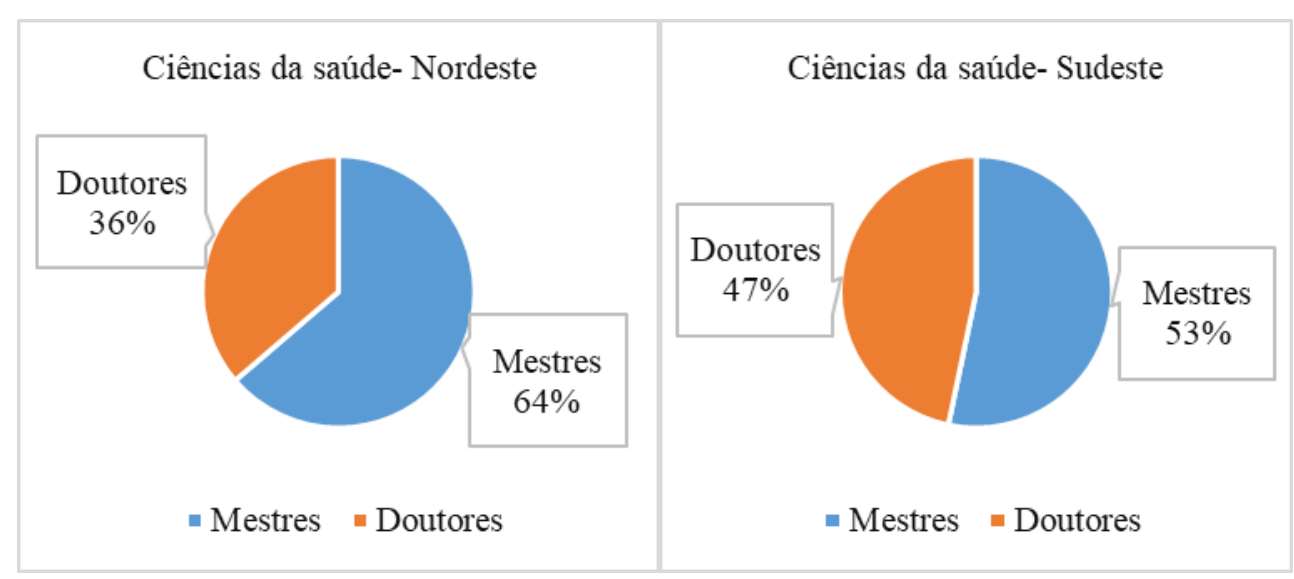

Fonte: CNPq (2021).

Gráfico 4. Produção cientifica das demais áreas cientificas no nordeste e sudeste. Teresina, PI, Brasil, 2021.
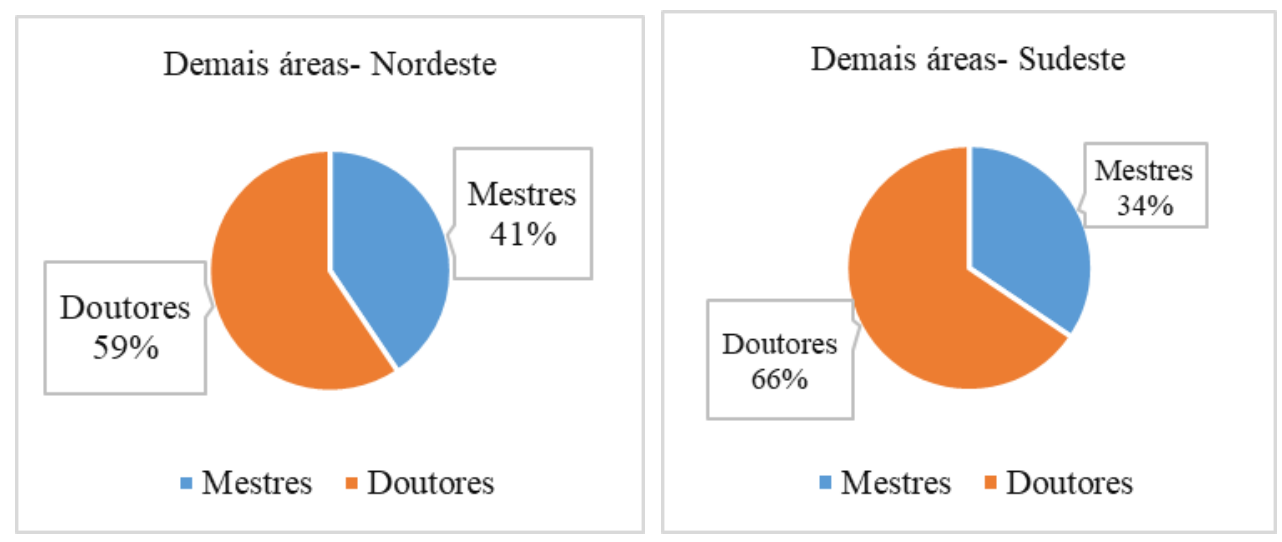

Fonte: CNPq (2021).

O Brasil enfrenta diversos desafios no tocante ao desenvolvimento e à consolidação de seu Sistema Nacional de Inovação. Dentre eles, destacam-se as necessidades de incentivo às atividades de Pesquisa e Desenvolvimento. Segundo o estudo de Magnin, Faria, Penteado e Takahashi (2020), foi feito uma análise onde os pesquisadores comprometidos com a pesquisa no cenário atual brasileiro, relataram sentirem-se em um "campo de batalha" onde é preciso lutar em todas as dimensões: pelo financiamento para a pesquisa, pela bolsa de iniciação científica, pelo parecer favorável à publicação, pelo interesse dos alunos, pelo tempo para ler e escrever artigos, para orientar discentes, elaborar projetos de pesquisa, atualizar os currículos, executar o financiamento eventualmente conquistado, realizar a prestação de contas, e assim por diante. Batalha-se até para se manter-se vinculado à pós-graduação.

O número de programas de mestrado continua crescendo a taxas significativas no Brasil. Em 1996, existiam 1.187 programas de mestrado no Brasil, enquanto que, no ano de 2014, o número desses programas já era de 3.620. É curioso perceber que apesar de a grande área das ciências da saúde ter sido a que apresentou, no período 1996-2014, a maior queda de participação relativa no número de programas de mestrado em geral, foi essa mesma grande área que também apresentou o maior ganho de pontos percentuais de participação relativa no número de programas de mestrado profissional (Magnin et al., 2020). Isso corrobora com os dados encontrado nessa pesquisa, e complementa o fato dos programas de mestrado e doutorado apresentar um número significativamente relevante, maior na região sudeste em comparação com a região nordeste exposto no Gráfico 1. 
Os programas de doutorado também vêm crescendo de forma significativa no Brasil, porém ainda é menor que os programas de mestrado. Também no caso dos doutorados, a grande área multidisciplinar apresentou um significativo crescimento nos 19 anos da série histórica. Isso pode ser explicado pelo fato que a região sudeste oferta maior número de programas de doutorados multidisciplinar, explicando o número expressivo na comparação das duas regiões, observado no Gráfico 1 (Bocorny et al., 2021). Além disso, as produções científicas seguem o princípio do maior número de participantes, mestrado ou doutorado, logo a produção científica é maior no grupo dos mestrados e na região sudeste, que já foi exposto anteriormente, observado no Gráfico 3.

É possível notar no Gráfico 2, que comparado com o Gráfico 1, as demais áreas têm um crescimento diferente. A concentração mais pronunciada é no Sudeste, que se destaca tanto no mestrado, como no doutorado. A região Sudeste é favorecida pela concentração de universidades e institutos de pesquisa historicamente consolidados (Suzigan \& Albuquerque, 2011), e pela maior disponibilidade de recursos humanos (Albuquerque et al., 2002) e financeiros, devido a políticas implementadas por importantes agências de fomento.

Estudos mostram que entre 2010-2016 a produção científica esteve mais forte nas áreas de medicina, física e química. Esse aumento da produção científica foi também observado em outras áreas como Odontologia, Saúde Pública e Fisioterapia (Martelli-Junior et al., 2010).

No Gráfico 4, temos dados em porcentagem dessas estatísticas, que mostram que a quantidade de doutores nas duas regiões é maior que a de mestres. Mecanismos contribuintes que podem ser citados, é a própria bolsa de produtividade em pesquisa que promove essa disputa entre, dando incentivo a formação de novos pesquisadores, como a publicação de novos artigos.

Os resultados da pesquisa demonstram claramente a existência de desigualdades no quantitativo da produção científica nas regiões. A pesquisa apresenta importante papel para a geração de novos conhecimentos.

Além disso, o estudo teve limitações, pois a plataforma Lattes não atualiza seus dados diariamente. Dessa forma, instiga-se novas pesquisas para acompanhar o progresso da quantidade de pesquisadores do Brasil e todo o tema relacionado.

\section{Conclusão}

A pesquisa apresenta importante papel para a geração de novos conhecimentos. O presente estudo apontou que o Brasil enfrenta diversos desafios no tocante ao desenvolvimento e à consolidação de seu Sistema Nacional de Inovação. Dentre eles, destacam-se as necessidades de incentivo às atividades de Pesquisa e Desenvolvimento. Os resultados da pesquisa demonstram claramente a existência de desigualdades no quantitativo da produção científica nas regiões.

Além disso, o estudo teve limitações, pois a plataforma Lattes não atualiza seus dados diariamente. Dessa forma, instiga-se novas pesquisas para acompanhar o progresso da quantidade de pesquisadores do Brasil e todo o tema relacionado.

\section{Referências}

Aaker, D.A., Kumar, V., \& Day, G.S. (2004). Pesquisa de marketing. Atlas.

Albuquerque, E. M., Simões, R., Baessa, A., Campolina, B., \& Silva, L. (2002). A Distribuição Espacial da Produção Científica e Tecnológica Brasileira: uma Descrição de Estatísticas de Produção Local de Patentes e Artigos Científicos. Revista Brasileira de Inovação, 1(2), 225-251. https://doi.org/10.20396/rbi.v1i2.8648860.

Barral, A., \& Barral-Netto, M. (2008). A Faculdade de Medicina da Bahia e a Ciência Médica. Gazeta Médica Da Bahia,78(1), 117-20. http://www.gmbahia.ufba.br/index.php/gmbahia/article/view/273.

Bocorny, A. E. P., Rebechi, R., Reppen, R., Delfino, M. C. N., \& Lameira, V. M. (2021). A produção de artigos da área das ciências da saúde com o auxílio de key lexical bundles: um estudo direcionado por corpus. DELTA: Documentação de Estudos Em Lingüística Teórica E Aplicada, 37(1), e2021370101. https://doi.org/10.1590/1678-460X2021370101. 
Research, Society and Development, v. 11, n. 3, e16611326334, 2022

(CC BY 4.0) | ISSN 2525-3409 | DOI: http://dx.doi.org/10.33448/rsd-v11i3.26334

Helene, A. F., \& Ribeiro, P. L. (2011). Brazilian scientific production, financial support, established investigators and doctoral graduates. Scientometrics, 89(2), 677-686. https://ideas.repec.org/a/spr/scient/v89y2011i2d10.1007_s11192-011-0470-2.html.

Magnin, L. S. L. T., Faria, J. H., Penteado, R. C., \& Takahashi, A. R. W. (2020). Produtivismo na pós-graduação em administração: posicionamentos dos pesquisadores brasileiros, estratégias de produção e desafios enfrentados. REAd. Revista Eletrônica de Administração (Porto Alegre), 26(2), 265-299. https://doi.org/10.1590/1413-2311.284.95633.

Martelli-Junior, H., Martelli, D. R. B., Quirino, I. G., Oliveira, M. C. L. A., Lima, L. S., \& Oliveira, E. A. (2010). Pesquisadores do CNPq na área de medicina: comparação das áreas de atuação. Revista Da Associação Médica Brasileira, 56(4), 478-483. https://doi.org/10.1590/S0104-42302010000400024.

Mattar, F.N. (2001). Pesquisa de marketing (3a ed.). Atlas.

Mendes, P. H. C., Martelli, D. R. B., Souza, W. P., Quirino Filho, S., \& Martelli Júnior, H. (2010). Perfil dos pesquisadores bolsistas de produtividade científica em medicina no CNPq, Brasil. Revista Brasileira de Educação Médica, 34(4), 535-541. https://doi.org/10.1590/S0100-55022010000400008.

Péret, A. C. A. P. A., \& Lima, M. L. R. (2003). A pesquisa e a formação do professor de Odontologia nas políticas internacionais e nacionais de educação. Revista Da ABENO, 3(2), 65-69. https://doi.org/10.30979/rev.abeno.v3i2.1459.

Sidone, O. J. G., Haddad, E. A., \& Mena-Chalco, J. P. (2016). A ciência nas regiões brasileiras: evolução da produção e das redes de colaboração científica. Transinformação, 28(1), 15-32. https://doi.org/10.1590/2318-08892016002800002.

Suzigan, W., \& Albuquerque, E. da M. e. (2011). The underestimated role of universities for the Brazilian system of innovation. Brazilian Journal of Political Economy, 31(1), 03-30. https://doi.org/10.1590/S0101-31572011000100001. 